\title{
Quantum Optimal Control Dynamics for Delay Intracellular and Multiple Chemotherapy Treatment (MCT) of Dual Delayed HIV - Pathogen Infections
}

\begin{abstract}
Bassey, E. Bassey ${ }^{*}$
*Department of Mathematics /Statistics, Cross River University of Technology, Calabar, Nigeria

*Corresponding Author: Bassey, E. Bassey, Department of Mathematics /Statistics, Cross River University of Technology, Calabar, Nigeria

Abstract: In furtherance to the pursuit for the advent of more précised and acceptable preventive and suppressive approach to the continual de-replication of viral load and parasitoid-pathogen with presupposed maximization of both $\mathrm{CD}^{+}$T-lymphocytes and cytotoxic T-lymphocytes (CTLs), this present study using ordinary differential equation, formulated a set of nonlinear complex 10-Dimensional mathematical dynamic dual HIV-pathogen model. In addition to the embedded dual infectivity, the novelty of this present work is in the incorporation of the crucial role of delay intracellular and immune effectors response in the presence of multiple chemotherapeutic treatments. Presenting the model as an optimal control problem and saddle with terminal time objective functional, classical Hamilton-Pontryagin function was explored in the analysis of derived quantum locally optimal algorithm of successive approximation for healthy CD4 ${ }^{+} T$ cells concentration. Using Runge-Kutter of order of precision 4 in a Mathcad surface, numerical validity of the model was conducted. Results of accompanying numerical simulations indicated the maximization of both healthy $\mathrm{CD}^{+}$T-lymphocytes and CTLs as a function of multiple chemotherapies with high toxicity and the presence of boosted immune effectors response under reduced systemic cost. Furthermore, maximal suppression of sensitive (infected T-cells and virions) state variables, which are daunted by persistent resistive infectious components suggests for a more articulated dual infectivity model.
\end{abstract}

Keywords: Dual-HIV-Pathogen-Infection, Delay-Intracellular, Multiple-Chemotherapy, Quantum-OptimalControl, Successive-Approximation, Sensitive-State-Variable, Resistive-State-Variable.

\section{INTRODUCTION}

Considered as the most dreaded transmittable infection with no known cogent medical cure, the human immunodeficiency virus (HIV), which often transmute to terminal irreversible manifestation - the acquired immunodeficiency syndrome (AIDS) have posed a challenging treat to the scientific world. The amiable quest of tackling this life threatening disease - HIV/AIDS infection has been through the application of mathematical modeling, which allows the utilization of significant knowledge of numerical methods for the optimization of host target immune system cells and the minimization of systemic cost, while suppressing viral victors below detectable clinical assay, see for examples the studies of models [14].

Furthermore, following the emerging new cases of multiplicity of HIV and its allies of pathogenic infections, it has become more unsafe and agreeably incomplete to mathematically and biological model eradication of HIV/AIDS infections without accounting for the epidemiological and biological behavior of the consequences of allying pathogenic infections. The seeming varying positive contributions from numerical methods in the dynamics of single strain HIV infection have considerably been extended to the diagnosis of the epidemiological and biological behaviors of dual HIV-pathogen infectivity as has been exemplified by models [5-9]. Consequentially, the varying results from these later models have been of immense importance in subduing the tenacity of the surging cases of dual viral infectivity.

Application of quantum locally optimal algorithm of successive approximation as a concept of numerical methods, which accounted for the mathematical simulations of human immune systems problems using varying optimization control strategies for immune processes and HI-virus infections were adequately 
discussed in the models [2, 10-15]. Of particular interest to this present paper, is the mathematical formulation by [16], which was based on the findings of model [11]. The model studied the numerical methods of optimal control of HIV-infection dynamics using quantum locally optimal algorithm of successive approximation technique. That model considered 7 - differential equations framed around 7 subgroups of the populations. The study investigated the role of numerical methods in solving complex problems in medicine and was able to define the lifespan of infected patients in terms of continuous and intermittent application of therapy. The methodological key components of the model involves the uninfected target cells $(T)$, infected sensitive cells $\left(T_{s}\right)$, infected cells resistant $\left(T_{r}\right)$, viral load sensitive $\left(V_{s}\right)$, viral load resistant $\left(V_{r}\right)$ and the introduced treatment functions $\eta_{1}(t)$ and $\eta_{2}(t)$. However, the above model lacks the finite biological attributive roles of delay intracellular and cell-mediated immune effectors response. Furthermore, the outcome of the result does not spelt the effects of allies of HIV infectivity.

Articulating the above seeming silent but unavoidable epidemiological constraints and motivated by the studies $[1,5]$, the present paper proposes and formulates using ordinary differential equations, an improved complex nonlinear 10-Dimensional dual HIV-pathogen dynamic delay-differential model, prime with the task of not only overcoming the lapses of model [16] but also to account for the methodological application of multiple chemotherapy treatment (MCT) in dual delay HIV-pathogen infections incorporated with delay intracellular and immune effectors response. Therefore, the present model is studied under the auspices of 10-subpopulations, which includes the critical roles of immune effectors response and two treatment functions $\eta_{1}(t)$ and $\eta_{2}(t)$. The methodology involves dual HIVpathogen infections on target cells distorted with reverse transcriptase inhibitors (RTI) and protease inhibitors (PIs) as treatment factors and clinically sandwich in two treatment discontinuation chambers denoted by $G_{n}=\left\{G_{s}, G_{r}\right\}$ with which the index - infection delay intracellular is accommodated. The variations of the present model with those of [5-9] are the incorporation of well-defined treatment functions $\eta_{1}(t)$ and $\eta_{2}(t)$ (on RTI and PIs). Moreso, the present model clearly articulates the mathematical representation of the biological attributes of the model state variables (to be defined in section 2).

Furthermore, to appreciate the critical functions of delay intracellular immune andeffectors response, we leap on the innovative ideas of the mathematical formulations studied by [1,5]. Thus, in the quantitative analysis of our complex nonlinear model to be presented as on optimal control problemand applying from the vast branches of numerical methods, we explore Hamilton-Pontryagin' function to establish the model quantum locally optimal algorithm for successive approximations. The approach of which had earlier been initiated in the vector of phase variables of [16].

The entire model is explicitly constituted into 6 sections with section 1 devoted to the introductory aspect. The material and methods adopted for this investigation, which involves the mathematical novelty of the study presented as problem statement model and transformed to an optimal control problem are develop in section 2. The analysis of the derived model is treated in section 3. Here, the model explores classical optimization technique known as Hamilton-Pontryagin's function in the evaluation of the system quantum locally optimal algorithm of successive approximations. Numerical simulations using in-built Runge-Kutter of order of precision 4 in a Mathcad surface and results of investigations are contained in section 4. Section 5 discusses the implications of the applied model and analyzes the obtained results of section 4. Finally, we draw in section 6, succinct conclusion and recommendations on the basis of achieved result. It is therefore anticipated that this present study will to a greater extent address the curiosity to overcome the curative impediment of dual HIV-pathogen infections.

\section{MATERIAL AND MethodS}

Here, we shall accomplish the intents of this section with crystal focus on presentation of mathematical problem statement of the model and the transformation of the derive model to an optimal control problem.

\subsection{Mathematical Problem Statement for Uncontrolled Treatment}

As a led way, we shall as highlighted in section 1; formulate the mathematical problem statement from the viewpoints of model $[1,5,8,16]$. From model [16], the study was formulated as a 7 - Dimensional vector of phase variables. The epidemiological derivation of the model was given as: 


$$
\begin{aligned}
\frac{d T(t)}{d t}=s_{1}-\frac{s_{2} V(t)}{B_{s}+V(t)}-\mu_{T} T(t)+\frac{\lambda_{1}}{C+V(t)} T(t) V(t)-\left(\eta_{1}(t) k_{s} V_{s}(t)+k_{r} V_{r}(t)\right) T(t), \\
\frac{d T_{s}(t)}{d t}=\eta_{1}(t) k_{s} V_{s}(t)-\mu_{T_{i}} T_{s}(t)-\frac{\lambda_{2}}{C_{i}+V(t)} T_{s}(t) V(t) \\
\frac{d T_{r}(t)}{d t}=k_{r} V_{r}(t) T(t)-\mu_{T_{i}} T_{r}(t)-\frac{\lambda_{2}}{C_{i}+V(t)} T_{r}(t) V(t), \\
\frac{d V_{s}(t)}{d t}=(1-q) \frac{\lambda_{3}}{C_{i}+V(t)} T_{s}(t) V(t)-k_{r} V_{s}(t) T(t)+\eta_{2}(t) \frac{G_{s} V_{s}}{B+V(t)} \\
\frac{d V_{r}(t)}{d t}=q \frac{\lambda_{3}}{C_{i}+V(t)} T_{s}(t) V(t)-k_{v} V_{r}(t) T(t)+G_{r}(V(t)) \frac{V_{r}}{B+V(t)} \\
\frac{d \eta_{1}(t)}{d t}=c_{1}\left(1-\eta_{1}(t)-u_{1}\right) \\
\frac{d \eta_{2}(t)}{d t}=\frac{c_{2}}{1-c_{3}}\left(1-\eta_{2}(t)-u_{2}\left(c_{2}-1\right)\right) .
\end{aligned}
$$

The epidemiological and biological descriptions of these state variables and parameters of model (1) will be reason out from the definition of our derived model (2) below. This becomes obvious in order to avoid the unnecessary repetition of definitions of some rerated biological parameters (functions).

Suppose the key components of the state variables for the present study are define as: $T_{u}$ - uninfected $\mathrm{CD}^{+} \mathrm{T}$ cells concentration, $T_{s}$ - infected $\mathrm{CD} 4^{+} \mathrm{T}$ cells sensitive to chemotherapy, $T_{r}$ - infected $\mathrm{CD} 4^{+} \mathrm{T}$ cells resistant to chemotherapy, $V_{s}$ - viral load sensitive to chemotherapy, $V_{r}$ - viral load resistive to chemotherapy, as was the case for model (1). Incorporated in this present model are $P_{s}$ - parasitoidpathogen sensitive to chemotherapy, $P_{r}$ - parasitoid-pathogen resistive to chemotherapy, $M_{E}$ - immune effectors response, $\eta_{1}$ - treatment function (i.e. RTI) on $T_{u}$ and $T_{s}$; and $\eta_{2}$ - treatment function (i.e. PIs) on $V_{s}$ and $P_{s}$. Then, we are bound to derive an improved complex nonlinear 10-Dimensional dual HIVpathogen dynamic delay-differential model. Now, from model (1) and invoking the innovative idea from [1] in relation to delay intracellular; the articulated critical role of cell-mediated immune effectors response as investigated by [5] in conjunction with the versatile knowledge of dual HIV-pathogen infectivity of model [8], we thus arrive at a modified and reformulated epidemiological and biological model governed by

$$
\begin{gathered}
\begin{array}{c}
\frac{d T_{u}(t)}{d t}=b_{1}-\frac{b_{2}\left(V_{T}(t)+P_{T}(t)\right)}{S_{T}+V_{T}(t)+P_{T}(t)}-\alpha_{1} T_{u}(t)+\frac{z_{u}}{S_{u}+V_{T}(t)+P_{T}(t)} T_{u}(t)\left[V_{T}(t)+P_{T}(t)\right] \\
-\left[\eta_{1}(t)\left(h_{s} V_{s}(t)+l_{s} P_{s}(t)\right)+\left(h_{r} V_{r}(t)+l_{r} P_{r}(t)\right)\right] T_{u}(t),
\end{array} \\
\begin{aligned}
\frac{d T_{s}(t)}{d t}= & \eta_{1}(t) e^{-\alpha_{2} \tau} T_{u}(t)\left[\left(h_{s} V_{s}(t)+l_{s} P_{s}(t)\right)\right]-\alpha_{2} T_{s}(t)
\end{aligned} \\
\quad+\frac{z_{s}}{S_{i}+V_{T}(t)+P_{T}(t)} T_{s}(t)\left[V_{T}(t)+P_{T}(t)\right]-\lambda M_{E}(t) T_{s}(t), \\
\frac{d T_{r}(t)}{d t}=\left[\left(h_{r} V_{r}(t)+l_{r} P_{r}(t)\right)\right]-\alpha_{2} T_{r}(t)-\frac{z_{s}}{S_{i}+V_{T}(t)+P_{T}(t)} T_{r}(t)\left[V_{T}(t)+P_{T}(t)\right], \\
\frac{d V_{s}(t)}{d t}=\left(1-q_{1}\right) \frac{z_{v}}{S_{v}+V_{T}(t)+P_{T}(t)} T_{s}(t)\left[V_{T}(t)+P_{T}(t)\right]-\mu_{v} V_{s}(t) T_{u}(t)+\eta_{2}(t) \frac{G_{s} V_{s}(t)}{S_{v}+V_{T}(t)+P_{T}(t)}, \\
\frac{d V_{r}(t)}{d t}=
\end{gathered}
$$




$$
\begin{gathered}
-\mu_{v} V_{r}(t) T_{u}(t)+G_{r}\left(V_{T}(t)+P_{T}(t)\right) \frac{V_{r}(t)}{S_{v}+V_{T}(t)+P_{T}(t)}, \\
\frac{d P_{s}(t)}{d t}=\left(1-q_{2}\right) \frac{z_{p}}{S_{i}+V_{T}(t)+P_{T}(t)} T_{s}(t)\left[V_{T}(t)+P_{T}(t)\right]-\mu_{p} P_{s}(t) T_{u}(t)+\eta_{2}(t) \frac{G_{s} P_{s}(t)}{S_{p}+V_{T}(t)+P_{T}(t)}, \\
\frac{d P_{r}(t)}{d t}=\frac{z_{p}}{S_{i}+V_{T}(t)+P_{T}(t)} T_{r}(t)\left[V_{T}(t)+P_{T}(t)\right]+q_{2} \frac{z_{p}}{S_{i}+V_{T}(t)+P_{T}(t)} T_{s}(t)\left[V_{T}(t)+P_{T}(t)\right] \\
\frac{d M_{E}(\mathrm{t})}{d t}=b_{M}+\frac{w_{M} T_{s}(t)}{T_{s}(t)+H_{w}} M_{E}-\frac{\delta_{M} T_{s}(t)}{T_{s}(t)+H_{\delta}} M_{E}-\mu_{M} M_{E}, \\
\frac{d \eta_{1}(t)}{d t}=\left(c_{1}+d_{1}\right)\left[1-\eta_{1}(t)-V_{1}(\mathrm{t})\right], \\
\frac{d \eta_{2}(t)}{d t}=\frac{c_{2}+d_{2}}{1-\left(c_{3}+d_{3}\right)}\left\{1-\eta_{2}(t)-r_{2}(t)\left(\left(c_{3}+d_{3}\right)-1\right)\right\},
\end{gathered}
$$

where $V_{s}(t)+V_{r}(t)=V_{T}(t)$ and $P_{s}(t)+P_{r}(t)=P_{T}(t)$. So that if we let $Q_{i}$ presents the model state variables, then $Q_{i}(0)=Q_{(i) 0}$ for all $t=t_{0}$.

Thus, equations of model (2) constitute the basic model with which we conduct the current present study. Unlike model (1), the innovative aspect of model (2) is reveal by the incorporation of sixth, seventh and eighth equations, which also have its biological contributive effects on other equations of the model. The epidemiological definitions of equations of model (2) and their mechanism of interactions can thus be given as follows: from the first equation, the term $b_{1}-\frac{b_{2}\left(V_{T}(t)+P_{T}(t)\right)}{S_{T}+V_{T}(t)+P_{T}(t)}$ is the source/proliferation of uninfected T-cells, $\alpha_{1} T_{u}(t)$ is the natural death rate of uninfected T-cells, $\frac{z_{u}}{S_{u}+V_{T}(t)+P_{T}(t)} T_{u}(t)\left[V_{T}(t)+P_{T}(t)\right]$ is the replication intensity of T-cells due to dual infections. This term plays crucial role in the immune system functioning by reacting to foreign matters (virions) ingress. Thus generating and increasing the concentration of immune effectors response. The last term of this equation $-\left[\eta_{1}(t)\left(h_{s} V_{s}(t)+l_{s} P_{s}(t)\right)+\left(h_{r} V_{r}(t)+l_{r} P_{r}(t)\right)\right] T_{u}(t)$ is the rate at which T-cells becomes infected and are cleared by treatment function $\eta_{1}(t)$.

From the second equation, since treatment does not affect resistive infected cells and resistive virions, the first term is the product of the exponential rate reflecting the death rate and time delay, which affects the inflow of infected T-cells by dual infectivity. The infected T-cells are cleared at the rate $\alpha_{2} T_{s}(t)$, while the dual viral infections sustain its presence by the term $\frac{z_{s}}{S_{i}+V_{T}(t)+P_{T}(t)} T_{s}(t)\left[V_{T}(t)+P_{T}(t)\right]$. The last term $\lambda M_{E}(t) T_{s}(t)$ is the loss rate of infected cells due to defense mechanism of immune effectors response.

In the third equation, the first term $\left[\left(h_{r} V_{r}(t)+l_{r} P_{r}(t)\right)\right]$ defines the inflow of resistive viral load in the immune system, while the term $\alpha_{2} T_{r}(t)$ denotes natural death rate of infected resistive T-cells. The sustainability of infected resistive T-cells following its replacement by sensitive infected cells is given by $\frac{z_{s}}{S_{i}+V_{T}(t)+P_{T}(t)} T_{r}(t)\left[V_{T}(t)+P_{T}(t)\right]$. 
The fourth and sixth equations having related terms represents the activities of the sensitive viral load and parasitoid-pathogen in the T-cells. Their respective first terms are the fractional replicative capacities of inflow of both viral load and pathogens into the infected T-cells, each having $\mu_{v} V_{s}(t) T_{u}(t)$ and $\mu_{p} P_{s}(t) T_{u}(t)$ loss rates. Their respective last terms are the discontinuation chambers of inflow of lymphoid sources of sensitive virions $\left(V_{s}, P_{s}\right)$ due to cohesive application of treatment function $\eta_{2}(t)$.

In a like manner, fifth and seventh equationsrepresenting resistive viral load and resistive pathogen can be describe along same biological behaviors. Their respective first and second terms are the sum of replicative rates of both resistive virions and fractional replicative capacities of sensitive virions that becomes resistive to chemotherapy. These resistive virions are loss at the rates $\mu_{v} V_{r}(t) T_{u}(t)$ and $\mu_{p} P_{r}(t) T_{u}(t)$, which are enhanced by the presence of discontinuation chambers as defined by their last terms.

From the eighth equation, we articulate the role of the immune effectors response. Here, the clearance rate of infected T-lymphocytes is a function of the immune effectors response (CTLs) with dynamics of $M_{E}$ adopted from the models $[5,17]$. The first term $b_{M}$ denotes the replication of more effector cells from co-existence of infected cells and immune effector cells interactions. The second term $\frac{w_{M} T_{s}(t)}{T_{s}(t)+H_{w}} M_{E}$ define the maximum birth rate of immune effectors in the presence of infected cells $T_{s}(t)$ and $M_{E}$; and having saturation birth rate $H_{w}$. The third term $\frac{\delta_{M} T_{s}(t)}{T_{s}(t)+H_{\delta}} M_{E}$ is the immune effectors maximum death rate with death saturation constant $H_{\delta}$, while the last term $\mu_{M} M_{E}$ describe immune effectors natural loss rate.

Furthermore, it is biologically obvious that when virions concentration in the blood plasma is large enough, defense mechanism transmutes information to the T-lymphocytes following chemotherapy application. Thus, sensitive virions are transmuted to resistive virions. This interactive process in model (2) is defined by the inclusion of the discontinuation function $G_{n}$, which is mathematically expressed as:

$$
G_{r}\left(V_{T}(t), P_{T}(t)\right)=\left\{\begin{array}{ccc}
0 & \text { for }_{T}, & P_{T}<V_{0}, P_{0} \\
G_{s} & \text { for } V_{T}, & P_{T} \geq V_{0}, P_{0}
\end{array}\right.
$$

where, $G_{r}$ is the discontinuation chambers of resistant virions replaced by sensitive virions following the administration of chemotherapy.

Drawing from model [11], where model [16] generated is basis, the treatment functions $\eta_{1}(t)$ and $\eta_{2}(t)$ are defined as function of time for single infection HI-virus. For model [11], which accounted for uncontrolled treatment i.e. the first five equations of model (1), the time function $\eta_{1}(t)$ and $\eta_{2}(t)$ were governed by

$$
\left.\begin{array}{c}
\eta_{1}(t)=\exp \left(-c_{1}\left(t-t_{0}\right)\right) \\
\eta_{2}(t)=\max \left\{\exp \left(-c_{2}\left(t-t_{0}\right)\right), c_{3}\right\}
\end{array}\right\}
$$

The model [16] introduced control variables $u_{1}(t)$ and $u_{2}(t)$ on treatment functions $\eta_{1}(t)$ and $\eta_{2}(t)$. The last two equations of model (1) clearly define the treatment function of model [16], which is derived as:

$$
\left.\begin{array}{l}
\frac{d \eta_{1}(t)}{d t}=c_{1}\left(1-\eta_{1}(t)-u_{1}\right) \\
\frac{d \eta_{2}(t)}{d t}=\frac{c_{2}}{1-c_{3}}\left(1-\eta_{2}(t)-u_{2}\left(c_{2}-1\right)\right)
\end{array}\right\}
$$


For dual HIV-pathogen virions as in the case of the present study, having parameter values as inscribed in table 2, the treatment functions is adequately describe by ninth and tenth equations of model (2). Therefore, from equations (4) and (5), the first functions defines the decreasing rate of T-cells infected by sensitive virions, while the second functions describe the effect of suppressed virions inflow in the blood plasma from lymphoid system. We must note that the resistive virions components are not affected by chemotherapy treatments.

Remarkably, the functions $\eta_{1}(t)$ and $\eta_{2}(t)$ defines the dynamic characters of treatment interactions processes, which accounts for intermittent treatment schedules in our present model. Epidemiologically, it becomes accommodating to introduce as in model (2), treatment control variables $r_{1}$ and $r_{2}$ denoting treatment switches. These variables are in the domain of $0 \leq r_{i=1,2} \leq 1$ such that if $r_{i=1,2}=1$, medication is eminent for infected patients (i.e. treatment is switch on) and if $r_{i=1,2}=0$, medication is discontinued (i.e. treatment is switch off).

Thus, in concretizing the validity of model (2); comparative test for equations (4) and (5) as well as the optimality system (to be determined in the later section), we establish a set of clinically compactible data for the state variables and parameters values as summarized in tables 1 and 2 below:

Table 1. Description of state variables with values for model (2)

\begin{tabular}{|c|c|c|c|}
\hline Variables & Dependent variables & Initial values & Units \\
\cline { 2 - 4 } & Description & 0.5 & \\
\hline$T_{u}$ & Uninfected T-lymph cells population & 0.2 & \\
$T_{s}$ & Infected CD4 ${ }^{+}$T cells sensitive to drug & 0.1 & \\
$V_{s}$ & Infected CD4 $4^{+}$T cells resistive to drug & 0.2 & \\
$V_{r}$ & Viral load sensitive to drug & 0.1 & \\
$P_{s}$ & Viral load resistive to drug & 0.1 & \\
$P_{r}$ & Pathogen sensitive to drug & 0.1 & \\
$M_{E}$ & Pathogen resistive to drug & 10 & \\
\hline$\eta_{1}$ & Immune effectors response & $\in[0,1]$ & \\
$\eta_{2}$ & Treatment function (RTI) on $T_{u}$ and $T_{s}$ & \\
\hline
\end{tabular}

Note: the table is a reflections of models $[1,8,16]$, clinically modified to meet the present novel model and compactible with RK4 software used in this investigation.

The corresponding model parameter values are defined by table 2 below:

Table 2. Summary of constants and parameter values of model (2)

\begin{tabular}{|c|c|c|c|}
\hline \multirow{2}{*}{$\begin{array}{c}\text { Parameter } \\
\text { symbols }\end{array}$} & Parameters and constants & \multirow[t]{2}{*}{ Values } & \multirow[t]{2}{*}{ Units } \\
\hline & Description & & \\
\hline 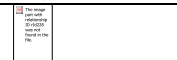 & Inflow of uninfected $\mathrm{CD} 4^{+} \mathrm{T}$ cells natural source & 0.8 & cu.mmd ${ }^{-1}$ \\
\hline$b_{2}$ & Proliferated uninfected $\mathrm{CD} 4^{+} \mathrm{T}$ cells inflow decrease & 0.4 & cu.mmd $d^{-1}$ \\
\hline$\alpha_{1}$ & Natural death rate of uninfected $\mathrm{CD} 4^{+} \mathrm{T}$ cells & 0.05 & $d^{-1}$ \\
\hline$\alpha_{2}$ & Natural death rate of infected $\mathrm{CD} 4^{+} \mathrm{T}$ cells & 0.02 & $d^{-1}$ \\
\hline$h_{s}$ & Rate $\mathrm{CD} 4^{+} \mathrm{T}$ cells becomes infected by $V_{s}$ & 0.005 & cu.mmd $d^{-1}$ \\
\hline$h_{r}$ & Rate $\mathrm{CD} 4^{+} \mathrm{T}$ cells becomes infected by $V_{r}$ & 0.005 & cu.mmd $d^{-1}$ \\
\hline$l_{s}$ & Rate $\mathrm{CD} 4^{+} \mathrm{T}$ cells becomes infected by $P_{s}$ & 0.002 & cu.mmd $d^{-1}$ \\
\hline$l_{r}$ & Rate $\mathrm{CD} 4^{+} \mathrm{T}$ cells becomes infected by $P_{r}$ & 0.002 & cu.mmd ${ }^{-1}$ \\
\hline$\mu_{v}$ & Viral load clearance rate by immune effectors response & 0.0062 & cu.mmd ${ }^{-1}$ \\
\hline
\end{tabular}


Quantum Optimal Control Dynamics for Delay Intracellular and Multiple Chemotherapy Treatment (MCT) of Dual Delayed HIV - Pathogen Infections

\begin{tabular}{|c|c|c|c|}
\hline$\mu_{p}$ & Pathogen clearance rate by immune effectors response & 0.0086 & cu.mmd ${ }^{-1}$ \\
\hline$z_{u}$ & Replication rate of uninfected $\mathrm{CD} 4^{+} \mathrm{T}$ cells & 0.025 & $d^{-1}$ \\
\hline$z_{v}$ & Replication rate of viral load & 0.008 & $d^{-1}$ \\
\hline$z_{p}$ & Replication rate of pathogen & 0.004 & $d^{-1}$ \\
\hline$z_{s}$ & Replication rate of infected $\mathrm{CD} 4^{+} \mathrm{T}$ cells & 0.025 & $d^{-1}$ \\
\hline$V_{0}$ & Viral load resistance threshold & 0.5 & cu.mm \\
\hline$P_{0}$ & Pathogen resistance threshold & 0.5 & cu.mm \\
\hline$q_{1}$ & Resistance viral load fraction due to replication capacity & 0.07 & \\
\hline$q_{2}$ & Resistance pathogen fraction due to replication capacity & 0.03 & \\
\hline$r_{i=1,2}$ & Treatment switches (control variables) & $r_{i} \in[0,1]$ & \\
\hline$S_{T}$ & Saturation coefficient of $\mathrm{CD} 4^{+} \mathrm{T}$ cells source & 0.38 & cu.mm $m^{-1}$ \\
\hline$S_{v}$ & Saturation coefficient of viral load external source & 0.20 & cu.mm $m^{-1}$ \\
\hline$S_{p}$ & Saturation coefficient of pathogen external source & 0.10 & cu.mm $m^{-1}$ \\
\hline$S_{u}$ & Saturation coefficient of uninfected $\mathrm{CD} 4^{+} \mathrm{T}$ cells source & 0.40 & cu.mm $m^{-1}$ \\
\hline$S_{i}$ & Saturation coefficient of infected $\mathrm{CD} 4^{+} \mathrm{T}$ cells source & 0.40 & cu.mm $m^{-1}$ \\
\hline$G_{s}$ & $\begin{array}{l}\text { Discontinuation chamber for external lymphoid source } \\
\text { of sensitive virions } V_{s} \text { and } P_{s}\end{array}$ & 0.42 & cu.mm $m^{-1}$ \\
\hline$G_{r}$ & $\begin{array}{l}\text { Discontinuation chamber for external lymphoid source } \\
\text { of resistive virions } V_{r} \text { and } P_{r}\end{array}$ & 0.42 & cu.mm $m^{-1}$ \\
\hline$c_{1}$ & Rate of suppression of viral infected $\mathrm{CD} 4^{+} \mathrm{T}$ cells by drug & 0.5 & \\
\hline$c_{2}$ & Rate of suppression of viral inflow from ext. lymphoid source by drug & 0.025 & \\
\hline$c_{3}$ & $\begin{array}{l}\text { maximum suppression of viral inflow from ext. lymphoid source by } \\
\text { drug }\end{array}$ & 0.15 & \\
\hline$d_{1}$ & Rate of suppression of pathogen infected $\mathrm{CD} 4^{+} \mathrm{T}$ cells by drug & 0.7 & \\
\hline$d_{2}$ & $\begin{array}{l}\text { Rate of suppression of pathogen inflow from ext. lymphoid source by } \\
\text { drug }\end{array}$ & 0.032 & \\
\hline$d_{3}$ & $\begin{array}{l}\text { maximum suppression of pathogen inflow from ext. lymphoid source } \\
\text { by drug }\end{array}$ & 0.25 & \\
\hline$\lambda$ & Rate of death of infected CD4+ T cells induced by immune effectors & 0.05 & cu.mmd ${ }^{-1}$ \\
\hline$w_{M}$ & Maximum birth rate of immune effectors & 0.03 & cu.mmd ${ }^{-1}$ \\
\hline$\delta_{M}$ & Maximum immune effected death rate & 0.02 & cu.mmd ${ }^{-1}$ \\
\hline$\mu_{M}$ & Death rate of CTLs effectors & 0.01 & $d^{-1}$ \\
\hline$\tau$ & Time delay & 0.5 & day \\
\hline$b_{M}$ & Immune effectors replication rate & 0.06 & cu.mmd $^{-1}$ \\
\hline$H_{w}$ & Immune effectors saturation birth rate & 10 & cu.mm $m^{-1}$ \\
\hline$H_{\delta}$ & Immune effectors saturation death rate & 30 & cu. $\mathrm{mm}^{-1}$ \\
\hline
\end{tabular}

Note: table 2 is a clinical modification of established parameter values of models [1, 8, 16]. 
According, the appreciation of the significance and slight variations (if any) of the treatment functions for the present study and those of models $[11,16]$ will be illustrated in section 4 .

\subsection{Statement of Optimal Control for MCT}

The introduction of optimization control strategy to treatment of infectious diseases (i.e. HIV and it allied pathogenic infections) is primarily hinge on the objective of prolongation of lifespan of infected patients following the application of prescribed chemotherapy. The measure by which the resulting outcome of an optimal control is accessed particularly for HIV infection is the quantitative level of healthy $\mathrm{CD} 4^{+} \mathrm{T}$ cell count concentration and the production of adaptive natural anti-HIV immune cytotoxic T-lymphocytes (CTLs) in relation to reduction in cost of treatment.

Therefore, in formulating the mathematical problem, which allows the investigation of the quantitative characteristics of these healthy T-cells and that of immune effectors response, the objective functional must be quantitatively formulated. For a study of model (2), having 10-Dimensional vectors phase space, the mathematical objective is considered in terms of its trajectories in a hypersurface bounds. From the study [18], the lowest severity category of T-cells concentration that leads to the development of AIDS ending with a lethal outcome is when viremia level is $T-$ cells $<200$ unit per cu.mm .

So that if we let this low level viremia bound be $T^{*}$, then the optimal problem of prolongation of patients lifespanis achievable if we set the bound on immune system to obtain

$$
T_{u}(t)-T^{*}=0 \text { for all } T^{*}=200 \text { per cu.mm } .
$$

This is to say that the objective functional $Q$, that define the optimization treatment problem is derive as:

$$
Q\left[r_{1}(t), r_{2}(t)\right]=t_{h}=\left\{t / T_{u}(t)=T^{*}\right\}
$$

for which the trajectory of model (2) attain hypersurface (6) at initial treatment of infection set-point. The value of $t_{h}$ in equation (7), is determined by the choice of treatment control functions $r_{1}(t)$ and $r_{2}(t)$. Hence, the numerical algorithm is geared towards establishing the functions for which equation (7) attains its maximum value for $r_{i}(t) \in[0,1], \mathrm{i}=1,2 \forall t \in\left[t_{i}, t_{h}\right]$, where $t_{i}$ is the initial set-point time.

Thus, equation (7) can be rewritten as:

$$
Q\left[r_{1}(t), r_{2}(t)\right]=t_{h}=\left\{t / T_{u}(t)=T^{*} \rightarrow \max _{r_{i}(t), i=1,2}\right\}
$$

Then we are to analyze the objective functional (8) to establish the quantum locally optimal algorithm of successive approximation in our next section.

\section{QUaNTUM LOCALly OPTIMAL AlgORITHM FOR DUAL HIV-PATHOGEN INFECTIVITY}

In this section, we shall derive the phase coordinates of model (2) using the control technique of optimization control method to establish the equation for successive increment functional and the quantum algorithm of control.

\subsection{Phase Coordinates for the System Model}

Using the phase conjugate, we shall explore the optimization method initiated by [19] to transform the 10Dimensional state variables to phase coordinates typical of model (2), i.e.

$$
f(t)=\left\{f_{T_{u}}, f_{T_{s}}, f_{T_{r}}, f_{V_{s}}, f_{V_{r}}, f_{P_{s}}, f_{P_{r}}, f_{M_{E}}, f_{\eta_{1}}, f_{\eta_{2}}\right\}
$$

and state the Hamilton-Pontryagin's function [20]

$$
\begin{aligned}
& H\left(T_{u}, T_{s}, T_{r}, V_{s}, V_{r}, P_{s}, P_{r}, M_{E}, \eta_{1}, \eta_{2},\right. \\
& \left.f_{T_{u}}, f_{T_{s}}, f_{T_{r}}, f_{V_{s}}, f_{V_{r}}, f_{P_{s}}, f_{P_{r}}, f_{M_{E}}, f_{\eta_{1}}, f_{\eta_{2}} r, r, t\right) \\
& \quad f_{T_{u}}\left\{b_{1}-\frac{b_{2}\left(V_{T}(t)+P_{T}(t)\right)}{S_{T}+V_{T}(t)+P_{T}(t)}-\alpha_{1} T_{u}(t)+\frac{z_{u}}{S_{u}+V_{T}(t)+P_{T}(t)} T_{u}(t)\left[V_{T}(t)+P_{T}(t)\right]\right. \\
& \left.\quad-\left[\eta_{1}(t)\left(h_{s} V_{s}(t)+l_{s} P_{s}(t)\right)+\left(h_{r} V_{r}(t)+l_{r} P_{r}(t)\right)\right] T_{u}(t)\right\}
\end{aligned}
$$




$$
\begin{aligned}
& +f_{T_{s}}\left\{\eta_{1} e^{-\alpha_{2} \tau}(t) T_{u}(t)\left[\left(h_{s} V_{s}(t)+l_{s} P_{s}(t)\right)+\left(h_{r} V_{r}(t)+l_{r} P_{r}(t)\right)\right]-\alpha_{2} T_{s}(t)\right. \\
& \left.+\frac{z_{s}}{S_{i}+V_{T}(t)+P_{T}(t)} T_{s}(t)\left[V_{T}(t)+P_{T}(t)\right]-\lambda M_{E}(t) T_{s}(t)\right\} \\
& +f_{T_{r}}\left\{\left[\left(h_{r} V_{r}(t)+l_{r} P_{r}(t)\right)\right] T_{u}(t)-\alpha_{2} T_{r}(t)+\frac{z_{s}}{S_{i}+V_{T}(t)+P_{T}(t)} T_{r}(t)\left[V_{T}(t)+P_{T}(t)\right]\right\} \\
& +f_{V_{s}}\left\{\left(1-q_{1}\right) \frac{z_{v}}{S_{i}+V_{T}(t)+P_{T}(t)} T_{s}(t)\left[V_{T}(t)+P_{T}(t)\right]-\mu_{v} T_{u}(t) V_{s}(t)+\eta_{2}(t) \frac{G_{s} V_{s}(t)}{S_{v}+V_{T}(t)+P_{T}(t)}\right\} \\
& +f_{V_{r}}\left\{\frac{z_{v}}{S_{i}+V_{T}(t)+P_{T}(t)} T_{r}(t)\left[V_{T}(t)+P_{T}(t)\right]+q_{1} \frac{z_{v}}{S_{i}+V_{T}(t)+P_{T}(t)} T_{r}(t)\left[V_{T}(t)+P_{T}(t)\right]\right. \\
& \left.-\mu_{v} T_{u}(t) V_{r}(t)+G_{r}\left(V_{T}(t)+P_{T}(t)\right) \frac{V_{r}(t)}{S_{v}+V_{T}(t)+P_{T}(t)}\right\} \\
& +f_{P_{s}}\left\{\left(1-q_{2}\right) \frac{z_{p}}{S_{i}+V_{T}(t)+P_{T}(t)} T_{s}(t)\left[V_{T}(t)+P_{T}(t)\right]-\mu_{p} T_{u}(t) V_{s}(t)+\eta_{2}(t) \frac{G_{s} P_{s}(t)}{S_{p}+V_{T}(t)+P_{T}(t)}\right\} \\
& +f_{P_{r}}\left\{\frac{z_{p}}{S_{i}+V_{T}(t)+P_{T}(t)} T_{r}(t)\left[V_{T}(t)+P_{T}(t)\right]+q_{2} \frac{z_{p}}{S_{i}+V_{T}(t)+P_{T}(t)} T_{s}(t)\left[V_{T}(t)+P_{T}(t)\right]\right. \\
& \left.-\mu_{p} T_{u}(t) P_{r}(t)+G_{r}\left(V_{T}(t)+P_{T}(t)\right) \frac{P_{r}(t)}{S_{p}+V_{T}(t)+P_{T}(t)}\right\} \\
& +f_{M_{E}}\left\{b_{M}+\frac{w_{M} T_{s}(t)}{T_{s}(t)+H_{w}} M_{E}-\frac{\delta_{M} T_{s}(t)}{T_{s}(t)+H_{\delta}} M_{E}-\mu_{M} M_{E}\right\} \\
& +f \eta_{1}\left\{\left(c_{1}+d_{1}\right)\left[1-\eta_{1}(t)-r_{1}(t)\right]\right\}+f \eta_{2}\left\{\frac{\left.c_{2}+d_{2}\right)}{1-\left(c_{3}+d_{3}\right)}\left[1-\eta_{2}(t)-r_{2}(t)\left(\left(c_{3}+d_{3}\right)-1\right)\right]\right\} \text {. }
\end{aligned}
$$

Next, we derive the corresponding system of delay-differential equations for the costate variables i.e.

$$
\begin{aligned}
& \frac{d f_{T_{u}}}{d t}=f_{T_{u}}\left[\alpha_{1}-\frac{z_{u}\left(V_{T}(t)+P_{T}(t)\right)}{S_{u}+V_{T}(t)+P_{T}(t)}+\eta_{1}(t)\left(h_{s} V_{s}(t)+l_{s} P_{s}(t)\right)+\left(h_{r} V_{r}(t)+l_{r} P_{r}(t)\right)\right] \\
& -f_{T_{s}}\left[\eta_{1}(t) e^{-\alpha_{2} \tau}\left(h_{s} V_{s}(t)+l_{s} P_{s}(t)\right)\right]-f_{T_{r}}\left[h_{r} V_{r}(t)+l_{r} P_{r}(t)\right]+f_{V_{s}}\left[\mu_{v} V_{s}(t)\right] \\
& +f_{V_{r}}\left[\mu_{v} V_{r}(t)\right]+f_{P_{s}}\left[\mu_{p} P_{s}(t)\right]+f_{P_{r}}\left[\mu_{p} P_{r}(t)\right] \\
& \frac{d f_{T_{s}}}{d t}=f_{T_{s}}\left[\alpha_{2}-\frac{z_{s}\left(V_{T}(t)+P_{T}(t)\right)}{S_{i}+V_{T}(t)+P_{T}(t)}+\lambda M_{E}(t)\right]-\frac{z_{v}\left(V_{T}(t)+P_{T}(t)\right)}{S_{i}+V_{T}(t)+P_{T}(t)}\left[f_{V_{s}}\left(1-q_{1}\right)+f_{V_{r}} q_{1}\right] \\
& -\frac{z_{p}\left(V_{T}(t)+P_{T}(t)\right)}{S_{i}+V_{T}(t)+P_{T}(t)}\left[f_{P_{s}}\left(1-q_{2}\right)+f_{p_{r}} q_{2}\right]-f_{M_{E}}\left[\frac{w_{M} M_{E}}{\left(T_{s}(t)+H_{w}\right)^{2}}+\frac{\delta_{M} M_{E}}{\left(T_{s}(t)+H_{\delta}\right)^{2}}\right], \\
& \frac{d f_{T_{r}}}{d t}=\frac{\left(V_{T}(t)+P_{T}(t)\right)}{S_{i}+V_{T}(t)+P_{T}(t)}\left[f_{T_{r}}\left(\alpha_{2}+z_{s}\right)+f_{V_{r}} z_{v}+f_{P_{r}} z_{p}\right] \\
& \frac{d f_{V_{s}}}{d t}=f_{T_{u}}\left[\frac{s_{u} P_{T}(t)}{\left(S_{T}+V_{T}(t)+P_{T}(t)\right)^{2}}\left(b_{2}-z_{u} T_{u}(t)\right)+\eta_{1}(t)\left(h_{s} T_{u}(t)\right)\right]-f_{T_{s}}\left[\eta_{1}(t) e^{-\alpha_{2} \tau} T_{u}(t) h_{s}\right] \\
& -\frac{z_{s}\left(S_{i} P_{T}(t)\right)}{\left(S_{i}+V_{T}(t)+P_{T}(t)\right)^{2}}\left[f_{T_{s}} T_{s}(t)+f_{T_{r}} T_{r}(t)\right] \\
& -\frac{z_{v}\left(S_{i} P_{T}(t)\right)}{\left(S_{i}+V_{T}(t)+P_{T}(t)\right)^{2}}\left[f_{V_{s}}\left(1-q_{1}\right) T_{s}(t)+f_{V_{r}}\left(T_{r}(t)+q_{1} T_{s}(t)\right)\right]+\mu_{r} T_{u}(t) \\
& -\frac{z_{p}\left(S_{i} P_{T}(t)\right)}{\left(S_{i}+V_{T}(t)+P_{T}(t)\right)^{2}}\left[f_{P_{s}}\left(1-q_{2}\right) T_{s}(t)+f_{P_{r}}\left(T_{r}(t)+q_{2} T_{s}(t)\right)\right]
\end{aligned}
$$




$$
\begin{aligned}
& -\frac{s_{v}}{\left(S_{v}+V_{T}(t)+P_{T}(t)\right)^{2}}\left[f_{V_{s}}\left(\eta_{1}(t) G_{s}\right)+f_{V_{r}}\left(G_{r} V_{r}(t) P_{T}(t)\right)\right] \\
& -\frac{s_{p}}{\left(S_{p}+V_{T}(t)+P_{T}(t)\right)^{2}}\left[f_{P_{s}}\left(\eta_{2}(t) G_{s} P_{s}(t) V_{T}(t)\right)+f_{P_{r}}\left(G_{r} P_{r}(t) P_{T}(t)\right)\right] \\
& \frac{d f_{V_{r}}}{d t}=f_{T_{u}}\left[\frac{b_{2}\left(s_{T} P_{T}(t)\right)}{\left(S_{T}+V_{T}(t)+P_{T}(t)\right)^{2}}-\frac{z_{u} T_{u}(t)\left(s_{u} P_{T}(t)\right)}{\left(S_{u}+V_{T}(t)+P_{T}(t)\right)^{2}}+h_{r} T_{u}(t)\right]-f_{T_{s}}\left[\eta_{1}(t) e^{-\alpha_{2} \tau} T_{u}(t) h_{s}\right] \\
& -\frac{z_{s}\left(s_{i} P_{T}(t)\right)}{\left(S_{i}+V_{T}(t)+P_{T}(t)\right)^{2}}\left[f_{T_{s}} T_{s}(t)+f_{T_{r}} T_{r}(t)\right]-f_{T_{r}}\left(h_{r} T_{u}(t)\right) \\
& -\frac{z_{v}\left(s_{i} P_{T}(t)\right)}{\left(S_{i}+V_{T}(t)+P_{T}(t)\right)^{2}}\left[f_{V_{s}}\left(1-q_{1}\right) T_{s}(t)+f_{V_{r}}\left(T_{r}(t)+q_{1} T_{s}(t)\right)\right]+f_{V_{r}}\left(\mu_{r} T_{u}(t)\right) \\
& -\frac{s_{v}}{\left(S_{v}+V_{T}(t)+P_{T}(t)\right)^{2}}\left[f_{V_{s}}\left(\eta_{1}(t) G_{s}\right)+f_{V_{r}}\left(G_{r} V_{r}(t) P_{T}(t)\right)\right] \\
& -\frac{z_{p}\left(S_{i} P_{T}(t)\right)}{\left(S_{i}+V_{T}(t)+P_{T}(t)\right)^{2}}\left[f_{P_{s}}\left(1-q_{2}\right) T_{s}(t)+f_{P_{r}}\left(T_{r}(t)+q_{2} T_{s}(t)\right)\right] \\
& -\frac{s_{p}}{\left(S_{p}+V_{T}(t)+P_{T}(t)\right)^{2}}\left[f_{P_{s}}\left(\eta_{2}(t) G_{s} P_{s}(t) V_{T}(t)\right)+f_{P_{r}}\left(G_{r}\left(V_{s}(t)+P_{r}(t)\right) P_{T}(t)\right)\right], \\
& \frac{d f_{P_{s}}}{d t}=f_{T_{u}}\left[\frac{s_{u} V_{T}(t)}{\left(S_{T}+V_{T}(t)+P_{T}(t)\right)^{2}}\left(b_{2}-z_{u} T_{u}(t)\right)+\eta_{1}(t)\left(l_{s} T_{u}(t)\right)\right] \\
& -f_{T_{s}}\left[\eta_{1}(t) e^{-\alpha_{2} \tau} T_{u}(t) l_{s}+\mathrm{z}_{s} T_{s}(t) \frac{s_{i} V_{T}(t)}{\left(S_{i}+V_{T}(t)+P_{T}(t)\right)^{2}}\right] \\
& -f_{T_{r}}\left[\frac{z_{s} T_{r}(t)\left(s_{i} V_{T}(t)\right)}{\left(S_{i}+V_{T}(t)+P_{T}(t)\right)^{2}}\right]-f_{V_{s}}\left[\frac{1}{\left(S_{i}+V_{T}(t)+P_{T}(t)\right)^{2}}\left(\left(1-q_{2}\right) z_{v} T_{s}(t)+\eta_{2}(t) G_{s} V_{s}(t) S_{v}\right)\right] \\
& -f_{V_{r}}\left[\frac{z_{v}\left(s_{i} V_{T}(t)\right)}{\left(S_{i}+V_{T}(t)+P_{T}(t)\right)^{2}}\left(T_{r}(t)+q_{1} T_{s}(t)\right)+G_{r} V_{r}(t) \frac{\left(s_{v} V_{T}(t)\right)}{\left(S_{v}+V_{T}(t)+P_{T}(t)\right)^{2}}\right] \\
& -f_{P_{s}}\left[\left(1-q_{2}\right) \frac{z_{p} T_{s}(t)\left(S_{i} P_{T}(t)\right)}{\left(S_{i}+V_{T}(t)+P_{T}(t)\right)^{2}}+\mu_{p} T_{u}(t)-\eta_{2}(t) \frac{G_{s} S_{p}}{\left(S_{p}+V_{T}(t)+P_{T}(t)\right)^{2}}\right] \\
& -f_{P_{r}}\left[\frac{z_{p}\left(S_{i} V_{T}(t)\right)}{\left(S_{p}+V_{T}(t)+P_{T}(t)\right)^{2}}\left(T_{r}(t)+q_{2} T_{s}(t)\right)+G_{r} \frac{P_{r}(t) S_{p} V_{T}(t)}{\left(S_{p}+V_{T}(t)+P_{T}(t)\right)^{2}}\right], \\
& \frac{d f_{P_{r}}}{d t}=f_{T_{u}}\left[\frac{b_{2}\left(S_{T} P_{T}(t)\right)}{\left(S_{T}+V_{T}(t)+P_{T}(t)\right)^{2}}-\frac{z_{u} T_{u}(t)\left(S_{u} P_{T}(t)\right)}{\left(S_{u}+V_{T}(t)+P_{T}(t)\right)^{2}}+l_{r} T_{u}(t)\right] \\
& -\frac{z_{s}\left(s_{i} V_{T}(t)\right)}{\left(S_{i}+V_{T}(t)+P_{T}(t)\right)^{2}}\left[f_{T_{s}} T_{s}(t)+f_{T_{r}} T_{r}(t)\right]-f_{T_{r}}\left(l_{r} T_{u}(t)\right) \\
& -f_{V_{s}}\left[\left(1-q_{1}\right) T_{s}(t) \frac{z_{v}\left(S_{i} V_{T}(t)\right)}{\left(S_{i}+V_{T}(t)+P_{T}(t)\right)^{2}}-\eta_{2}(t) G_{r} \frac{V_{s}(t) S_{v} P_{T}(t)}{\left(S_{p}+V_{T}(t)+P_{T}(t)\right)^{2}}\right] \\
& -f_{V_{r}}\left[\frac{z_{v} T_{r}(t)\left(S_{i} V_{T}(t)\right)}{\left(S_{i}+V_{T}(t)+P_{T}(t)\right)^{2}}\left(1-q_{1}\right)+G_{r}\left(V_{T}(t)+P_{T}(t)\right) \frac{V_{r}(t) S_{v}}{\left(S_{v}+V_{T}(t)+P_{T}(t)\right)^{2}}\right] \\
& -f_{P_{s}}\left[\left(1-q_{2}\right) \frac{z_{p} T_{s}(t)\left(S_{i} V_{T}(t)\right)}{\left(S_{i}+V_{T}(t)+P_{T}(t)\right)^{2}}-\eta_{2}(t) G_{s} \frac{P_{s}(t) S_{p} P_{T}(t)}{\left(S_{p}+V_{T}(t)+P_{T}(t)\right)^{2}}\right]
\end{aligned}
$$




$$
\begin{gathered}
-f_{P_{r}}\left[\frac{z_{p} S_{i} V_{T}(t)}{\left(S_{p}+V_{T}(t)+P_{T}(t)\right)^{2}}\left(T_{r}(t)+q_{2} T_{s}(t)\right)-\mu_{p} T_{u}(t)+G_{r}\left(V_{T}(t)+P_{T}(t)\right) \frac{V_{p}(t) P_{T}(t)}{\left(S_{p}+V_{T}(t)+P_{T}(t)\right)^{2}}\right], \\
\frac{d f_{M_{E}}}{d t}=f_{T_{s}}\left(-\lambda T_{s}(t)\right)-f_{M_{E}}\left(\frac{w_{M} M_{E} H_{w}}{\left(T_{s}(t)+H_{w}\right)^{2}}+\frac{\delta_{M} M_{E} H_{\delta}}{\left(T_{s}(t)+H_{\delta}\right)^{2}}\right)-f_{M_{E}}(\mu \mathrm{M}), \\
\frac{d f \eta_{1}}{d t}=f_{T_{u}}\left[h_{s} V_{s}(t)+l_{s} P_{s}(t)\right]-f_{T_{s}}\left(T_{u}(t)\left(h_{s} V_{s}(t)+l_{s} P_{s}(t)\right) e^{-\alpha_{2} \tau}\right)-f \eta_{1}\left(c_{1}+d_{1}\right) \\
\frac{d f \eta_{2}}{d t}=f_{V_{s}}\left[\frac{G_{s} V_{s}(t)}{S_{v}+V_{T}(t)+P_{T}(t)}\right]-f_{P_{s}}\left[\frac{G_{s} P_{s}(t)}{S_{p}+V_{T}(t)+P_{T}(t)}\right]-f \eta_{2}\left(\frac{\left.c_{2}+d_{2}\right)}{1-\left(c_{3}+d_{3}\right)}\right) .
\end{gathered}
$$

Equations (10) and (11) indicate that with the application of the optimization methods of [16, 19], we can solve the maximization problem of equation (7) by successive increase in the values of the functional $Q$ of equation (6), based on solutions of model (2) and costate equation (11). Therefore, the boundary conditions on costate variable at the right side of the trajectory are given as:

$$
\begin{gathered}
f_{T_{u}}\left(t_{0}\right)=-\left(\left.\frac{d T_{u}}{d t}\right|_{t=t_{0}}\right)^{-1}, \\
f_{T_{s}}\left(t_{0}\right)=f_{T_{r}}\left(t_{0}\right)=f_{V_{s}}\left(t_{0}\right)=f_{V_{r}}\left(t_{0}\right)=f_{P_{s}}\left(t_{0}\right)=f_{P_{r}}\left(t_{0}\right)=f_{M_{E}}\left(t_{0}\right)=f_{\eta_{1}}\left(t_{0}\right)=f_{\eta_{2}}\left(t_{0}\right)=0
\end{gathered}
$$

and which corresponds to the problem of the maximization of the functional (7) on hypersurface (6). Furthermore, it is observed that due to the discontinuation function (3), the right hand side of model (2) makes sharp changes when the trajectory passes across the hypersurface

$$
\left.\begin{array}{l}
\Omega_{1}\left(V_{s}, V_{r}\right)=V_{s}+V_{r}-V_{0}=0 \\
\Omega_{2}\left(P_{s}, P_{r}\right)=P_{s}+P_{r}-V_{0}=0
\end{array}\right\}
$$

Then, it can be said that once the trajectories model (2) passes hypersurface (13), the costate variable vector-valued function (9) satisfies the jump conditions [19]

$$
\begin{gathered}
f_{V_{s}}(t-0)=f_{V_{s}}(t+0)-v \frac{\partial \Omega_{1}}{\partial V_{s}}=f_{V_{s}}(t+0)-v, \\
f_{V_{r}}(t-0)=f_{V_{s}}(t+0)-v \frac{\partial \Omega_{1}}{\partial V_{r}}=f_{V_{r}}(t+0)-v, \\
f_{p_{s}}(t-0)=f_{p_{s}}(t+0)-p \frac{\partial \Omega_{2}}{\partial P_{s}}=f_{p_{s}}(t+0)-p, \\
f_{p_{r}}(t-0)=f_{p_{r}}(t+0)-p \frac{\partial \Omega_{2}}{\partial P_{r}}=f_{p_{r}}(t+0)-p .
\end{gathered}
$$

The corresponding coefficients of jump $v$ and $p$ are determined by the expressions

$$
v=\frac{f_{v_{r}}(t+0) \frac{G_{s} V_{r}(t)}{S_{V}+V_{T}(t)+P_{T}(t)} \operatorname{sign}\left[V_{s}(t-0)+V_{r}(t-0)-V_{0}\right]}{g_{v_{s}}^{-1}[(x(t), r(t), t)]+g_{v_{r}}^{-1}[(x(t), r(t), t)]}
$$

and

$$
p=\frac{f_{p_{r}}(t+0) \frac{G_{s} P_{r}(t)}{S_{P}+V_{T}(t)+P_{T}(t)} \operatorname{sign}\left[P_{s}(t-0)+P_{r}(t-0)-P_{0}\right]}{q_{p_{s}}^{-1}[(x(t), r(t), t)]+q_{p_{r}}^{-1}[(x(t), r(t), t)]} .
$$

From equation (15), the functions $g_{v_{s}}^{-1}, g_{v_{r}}^{-1}, q_{p_{s}}^{-1}$ and $q_{p_{r}}^{-1}$ are the right-hand sides of the fourth-seventh equations of hypersurface (13). 


\subsection{Equation for Successive Increment Functional}

For a specific optimal control problem, one approach of resolving the problem of healthy $\mathrm{CD}^{+} \mathrm{T}-$ lymphocytes maximization is the process of successive increment of control functional constructed as follows: Let the control functions be $r_{1}=r_{1}(t)$ and $r_{2}=r_{2}(t)$, then we can solve the Cauchy problem for model (2) having initial conditions as specified in model (2). Then, the trajectory of the control functional is obtained as:

$$
x(t)=\left\{T_{u}(t), T_{s}(t), T(t), V_{s}(t), V_{r}(t), P_{s}(t), P_{r}(t), M_{E}(t), \eta_{1}(t), \eta_{2}(t)\right\}, \forall t_{0} \leq t \leq t_{f}
$$

is determined in conjunction with the values of functional (7) on the supersurface (6). Therefore, we present the functional (7) with changed control functions i.e. $r_{1 c}=r_{1}(t)+\Delta r_{1}(t)$ and $r_{2 c}=r_{2}(t)+\Delta r_{2}(t)$. Then equation (7) can be rewritten as:

$$
Q\left[r_{1}(t)+\Delta r_{1}(t), r_{2}(t)+\Delta r_{2}(t)\right]
$$

The variation of functional (17) and (7) is thus:

$$
\Delta Q=Q\left[r_{1}(t)+\Delta r_{1}(t), r_{2}(t)+\Delta r_{2}(t)\right]-Q\left[r_{1}(t), r_{2}(t)\right],
$$

which is computed using equations (11)-(15) for costate variables.

Commutatively, if we let $r_{1}=r_{1}(t)$ and $r_{2}=r_{2}(t)$ in equation (10) and its trajectory corresponding to the right-hand side of equation (11) such that we integrate using boundary conditions (12) and accounting for equations (14) and (15) with reversed time. Then the vector-valued functional of costate variables to be computed are:

$$
f(t)=\left\{f_{T_{u}}(t), f_{T_{s}}(t), f_{T_{r}}(t), f_{V_{s}}(t), f_{V_{r}}(t), f_{P_{s}}(t), f_{P_{r}}(t), f_{M_{E}}(t), f_{\eta_{1}}(t), f_{\eta_{2}}(t)\right\}, \forall t_{0} \leq t \leq t_{f}
$$

In this considered problem, the change in functional (12), which is determined with the aid of equations (16) and (19), is obtained as follows:

$$
\Delta Q=\Delta Q_{1}+\Delta Q_{2}+0\left[\Delta Q_{1}+\Delta Q_{2}\right]
$$

where

$$
\left.\begin{array}{c}
\Delta Q_{1}=-\int_{t_{0}}^{t_{f}}\left[\left(c_{1}+d_{1}\right) \eta_{1}(t)+\Delta r_{1}(t)\right] d t \\
\Delta Q_{2}=-\int_{t_{0}}^{t_{f}}\left[\left(c_{2}+d_{2}\right) \eta_{2}(t)+\Delta r_{2}(t)\right] d t
\end{array}\right\}
$$

with $0\left[\Delta Q_{1}+\Delta Q_{2}\right]$ as the system error, determined by a higher order infinitesimal than $\Delta Q_{1}$ and $\Delta Q_{2}$.

Of note, is the fact that for a nonlinear delay-differential equation as model (2), the method of successive increment of control measure is achieved provided the following two conditions are satisfied: i) - the trajectory iteration is superimposed by any external source, ii) - the change in the control measure values are sufficiently small. The proof is standard and could be found in $[4,9]$.

\subsection{Quantum Locally Optimal Algorithm}

A satisfactory application of equation (20) in simulation of successive increment control requires the clinical conduct of both the choice of magnitude of the change controls and the rational localization of these changes. Condition (ii) above is provided by the localized small time interval i.e. $\Delta t_{i=1,2}$ such that the magnitude of the changes in $\Delta Q_{i=1,2}$ is proportional to the control quanta defined by

$$
\Delta R_{1}=\Delta r_{1} \Delta t_{1} ; \Delta R_{2}=\Delta r_{2} \Delta t_{2} .
$$

If we then substitute the above control quanta (22) into equation (21), the resulting control quanta become:

$$
\left.\begin{array}{c}
\Delta Q_{1}=-\left(c_{1}+d_{1}\right) f \eta_{1}\left(t_{1}\right) \Delta R_{1}, \\
\Delta Q_{2}=-\left(c_{2}+d_{2}\right) f \eta_{2}\left(t_{2}\right) \Delta R_{2}
\end{array}\right\}
$$


The control quanta (23) is a clear indication of the fact that the magnitudes of changes in $\Delta Q_{i=1,2}$ for a given fixed values of quanta (13) can be determined by the values of treatment functions $\eta_{1}(t)$ and $\eta_{2}(t)$ for a time instants $t_{1}$ and $t_{2}$, achieved by the introduction of control quanta (22).

Remarkably, it is convenient that the time instants for iterations satisfies the condition

$$
\left.\begin{array}{rl}
\Delta Q_{1} & =\max \left[-\left(c_{1}+d_{1}\right) f \eta_{1}\left(t_{1}\right) \Delta R_{1}\right], \\
\Delta Q_{2} & =\max \left[-\left(c_{2}+d_{2}\right) f \eta_{2}\left(t_{2}\right) \Delta R_{2}\right]
\end{array}\right\} .
$$

Condition (24) is applicable if we explicitly defined $\Delta R_{1}$ and $\Delta R_{2}$ taken from quanta (22) with the help of the following notations:

$$
\begin{gathered}
D_{1}=\max _{t \in\left[t_{i}, t_{h}\right]}\left[\left(c_{1}+d_{1}\right) f \eta_{1}(t)\left(2 r_{1}(t)-1\right]\right. \\
t_{1}=\underset{t \in\left[t_{i}, t_{h}\right]}{\arg \max }\left[\left(c_{1}+d_{1}\right) f \eta_{1}(t)\left(2 r_{1}(t)-1\right],\right.
\end{gathered}
$$

and

$$
\begin{gathered}
D_{2}=\max _{t \in\left[t_{i}, t_{h}\right]}\left[\left(c_{2}+d_{2}\right) f \eta_{2}(t)\left(2 r_{2}(t)-1\right]\right. \\
t_{2}=\underset{t \in\left[t_{i}, t_{h}\right]}{\arg \max _{h}\left[\left(c_{2}+d_{2}\right) f \eta_{2}(t)\left(2 r_{2}(t)-1\right]\right.} .
\end{gathered}
$$

Then, the validity of condition (24) is determined by the equations for $\Delta R_{i}, i=1,2$ :

$$
\begin{aligned}
\Delta R_{1} & =\left\{\begin{array}{c}
{\left[1-2 r_{1}\left(t_{1}\right)\right] \Delta t_{1}, \text { if } D_{1}>0} \\
0, \text { otherwise; }
\end{array}\right. \\
\Delta R_{2} & =\left\{\begin{array}{c}
{\left[1-2 r_{2}\left(t_{2}\right)\right] \Delta t_{2}, \text { if } D_{2}>0} \\
0, \text { otherwise }
\end{array} .\right.
\end{aligned}
$$

Available computational case study, which agree with the present study (but for a single HIV infection), is provided by [16]. The result of the experiment indicates that consistent convergence of successive iteration requires that control quanta (22) should necessary satisfy the inequalities: $\left|\Delta R_{1}\right| \leq 1 / 5$ and $\left|\Delta R_{2}\right| \leq 1 / 5$ with values of $t_{i}, i=1,2$ chosen from the basis of integration of model (2) and equation (4).The implication from equation (25) is that for each iteration of $1 / 5$ a day, a decision for drug administration should be taken in favor of either of the following three alternatives: i) treatment is unaltered and is continuous; ii) treatment is to be altered/change; and iii) treatment to be completely discontinued.

\section{NUMERICAL ILLUSTRATIONS}

A number of maximal illustrations to cover a range of equations derived are conducted in this section. First, we're required to draw the importance of this present study by simulating a comparative view of the treatment functions of this present investigation and those of related prior models by $[11,16]$. Using inbuilt Runge-Kutter of order of precision 4 in Mathcad surface, we at once simulate these functions as presented by fig. 1(a-c) below. For the compatibility of state components with software, the state variables are converted to read:

$$
\left\{T_{u}(t), T_{s}(t), T(t){ }_{r}, V_{s}(t), V_{r}(t), P_{s}(t), P_{r}(t), M_{E}(t), \eta_{1}(t), \eta_{2}(t)\right\}=\sum_{i=1}^{10} H_{i}
$$

such that $\sum_{i=1}^{10} H_{i}=\left(H_{1} \cdots \cdots \cdots H_{10}\right)^{T}$ with values as contains in table 1 and parameter values in table 2 respectively. From models $[11,16]$ we have $t_{0}=0, t_{f}=50, c_{1}=0.5, c_{2}=0,025, c_{3}=0,15$. Then the graphical simulations are as follows: 

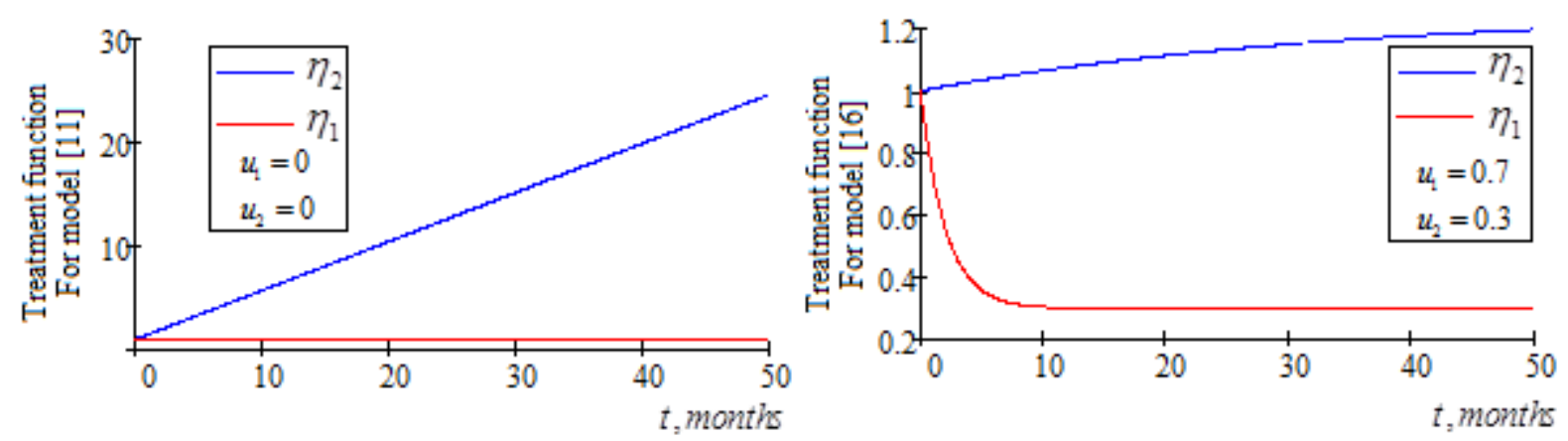

a) Simulation of treatment function $\eta_{1}=0=\eta_{2}$ b) Simulation of treatment function $\eta_{1}=1=\eta_{2}$

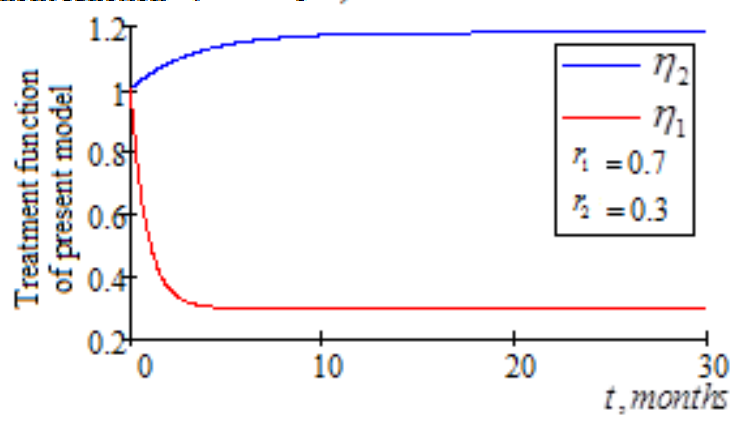

c) Simulation of treatment function $\eta_{1}=1=\eta_{2}$

Fig. 1(a-c) Comparative simulations of treatment

Functions for models 11,16] and present model

From the graphical view of fig. 1(a) representing [11], the function $\eta_{1}(t)$ having $1.0 \leq \eta_{1}(t) \leq 1.2 \times 10^{-5}$ for $t_{f} \leq 50$ months, reflect the gradual decrease in the last term of first equation of model (1). This explains the rate of effect of sensitive virions on infected T-lymphocytes. The second function $\eta_{2}(t)$ having $1.0 \leq \eta_{2}(t) \leq 24.618$ for $t_{f} \leq 50$ months represent the effect of suppression of virions inflow into the blood plasma.

In model (1), this circumstance is define and treated by sixth and seventh equations and in our present model (2), equations ninth and tenth adequately define the dual treatment functions. In both later models, two treatment control variables are introduced with domain 0 and 1 . The treatment function of model (1) is illustrated as in fig. 1(b) above [16]. The first function of fig. 1(b) exhibits sharp concave declination at the first 3 months with value $\eta_{1}(t)=0.3$ and attain stability thereafter and for the duration of investigation. The second function $\eta_{2}(t)$ exhibit slight linear inclination with value $\eta_{2}(t) \leq 1.196$ for $t_{f} \leq 50$ months.

In our present model (2), we illustrate the treatment function as presented by fig. 1(c). Here, the first function $\eta_{1}(t)$ exhibit sharp reduction for drug required i.e. $\eta_{1}(\mathrm{t}) \leq 0.3$ for treatment of infected $\mathrm{T}$ lymphocytes at the $2^{\text {nd }}$ month and then assume stability for the rest of investigation interval. The second function $\eta_{2}(t)$ exhibits initial small increase and then attain stability in the amount of chemotherapy required for the suppression of virions i.e. $\eta_{2}(t) \leq 1.18$ for $t_{f} \leq 30$ months. Of note, fig. 1 (b and c) demonstrate similar behavior in their treatment functions with that of present model exhibiting a more improve reduced amount of required therapy when compared with those of previous models.

Moving further, we consider the case of untreated dual infectivity as presented by fig. 2 (a-c). 

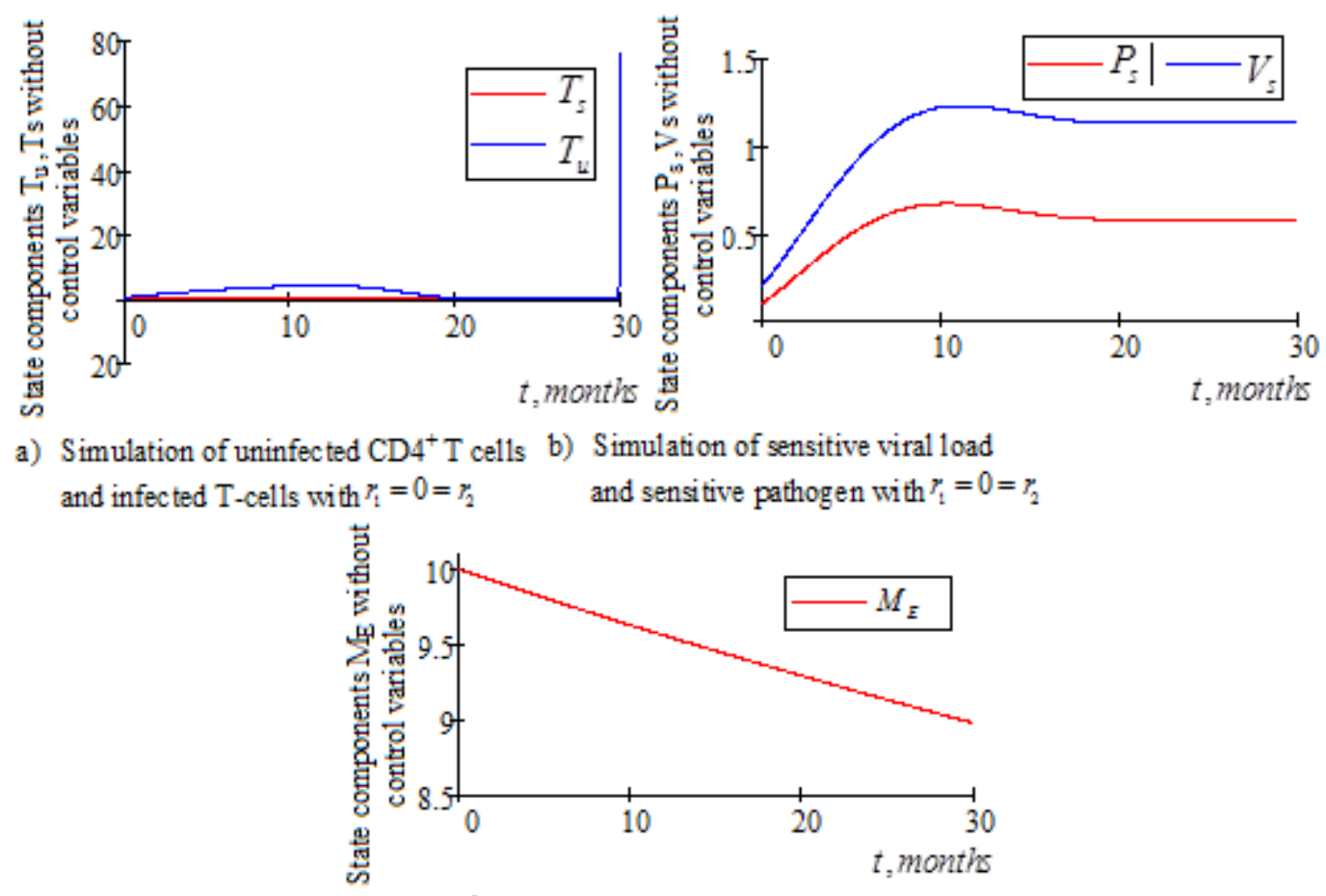

c) Simulation of immune effectors response with $r_{1}=0=r_{2}$

Fig. 2(a-c)Simulation dynamics of untreated dual delay HIV-pathogen model with $r_{1}=0=r_{2}$

Clearly, fig. 2(a-c) above illustrates the biological behaviors of untreated dual HIV-pathogen infections, which is subjected to only the critical role of CTLs. Sustaining the values of tables $(1 \& 2)$, fig. 2(a) represent the numerical integration of uninfected T- cells and infected sensitive T-cells. The function $T_{u}(t)$ exhibits gradual initial increase having sustained population of $0.5 \leq T_{u}(t) \leq 7.02$ unit / cu.mm after set-point for $t_{f} \leq 10$ months of infection period. This outcome is attributed to the initial defense established by the immune effectors response. $T_{u}(t)$ declinedsubsequently due to consistent replication of dual virions and infected T-cells. Uninfected T-cells thus decline to $T_{u}(t)=3.85 \times 10^{-3}$ unit / cu.mm after 20 months. $\mathrm{CD} 4^{+} \mathrm{T}$ cells are seen to increase again at the $30^{\text {th }}$ month of investigation, which signifies the sudden rebounds of CTLs. The second function $T_{S}(t)$, diminished in trajectory and is a consequence of the critical role of CTLs defense mechanism such that $0.025 \leq T_{s}(t) \leq 0.2$ for $t_{f} \leq 3$ months and remain at near zero for the rest of investigation period.

For fig. 2 (b), viral load under no chemotherapy and with rapid rate of viral load replication result to continuous viral load increase from $0.2 \rightarrow 1.233$ for $t_{f} \leq 10$ months and then decline to stability with $V_{s}(t)=0.6$ unit / cu.mm after $t_{f} \leq 18$ months and through duration of investigation. The second function $P_{s}(t)$ exhibit similar behavior with increasing value $0.1 \leq P_{s}(t) \leq 0.64$ for $t_{f} \leq 10$ months. Infectious pathogen attains stability at $P_{s}(t) \leq 0.58$ for $t_{f} \geq 18$ months. Fig. 2(c) demonstrates the efficacy of the immune effectors response in the absence of any other treatment factors. Of interest, CTLs always remain positive following the natural replicative ability, which is a function of virions concentration. The reduction in CTLs is due to its clearance rate of virions and possible natural death i.e. $8.983 \leq M_{E}(t) \leq 10$ for $t_{f} \leq 30$ months.

For the case of treatment application, we simulates as in fig. 3(a-c) below: 

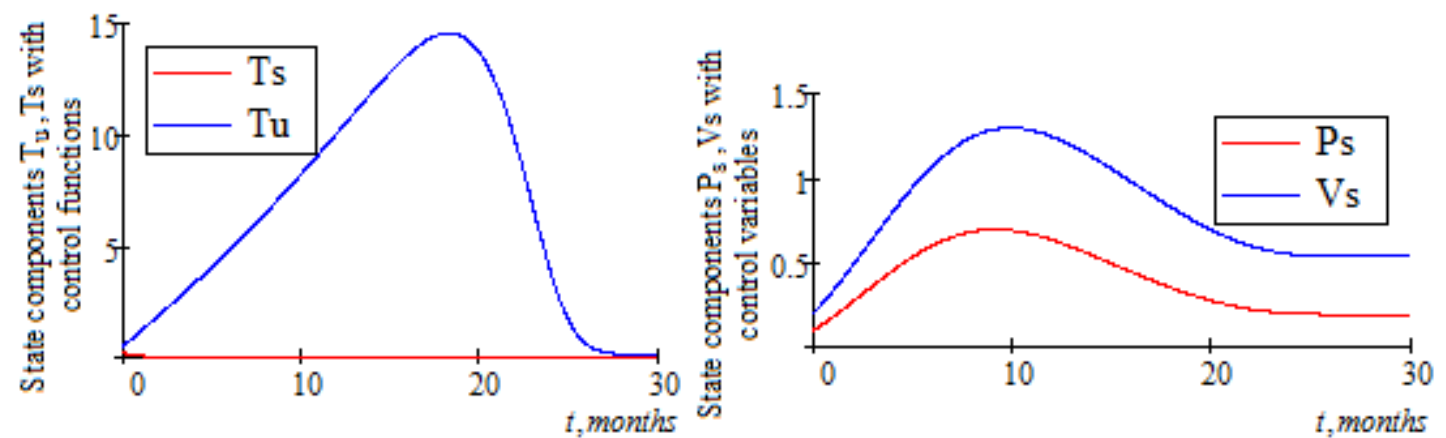

a) Simulation of uninfected $\mathrm{CD} 4^{+} \mathrm{T}$ cells and infected T-cells with $r_{1}=0.7, r_{2}=0.3$

b) Simulation of sensitive viral load and

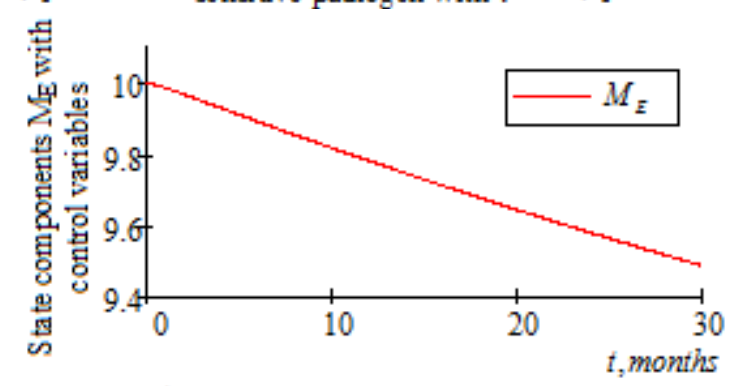

c) Simulation of immune effectors response with $r_{1}=0.7, r_{2}=0.3$

Fig. 3(a-c) Simulation dynamics of optimal treatmentregiments of dual delay HIV-pathogenmodel with $r_{1}=0.7, r_{2}=0.3$

Here, sustaining state and parameters values of tables $(1 \& 2)$, with slight variations i.e.

$$
\begin{aligned}
& z_{u}=0.045, z_{v}=0.004, z_{p}=0.002, h_{s}=0.005, h_{s}=0.005, \\
& h_{r}=0.005, l_{s}=0.002, l_{r}=0.002, w M=0.05, b_{M}=0.005,
\end{aligned}
$$

which is due to induced treatment factors, the following results were achieved: in fig. 3(a), the function $T_{u}(t)$ exhibits accelerated trajectory parabola in healthy $\mathrm{CD} 4^{+} \mathrm{T}$ cells with value $0.5 \leq T_{u}(t) \leq 14.569$ unit / cu.mm maximal for $t_{f} \leq 12$ months. $\mathrm{CD}^{+} \mathrm{T}$ cells thereafter decline to stability of $T_{u}(t) \leq 0.056$ unit / cu.mm at $27 \leq t_{f} \leq 30$ months. The second function $T_{s}(t)$ indicates sharp and rapid decline after 3 months of cohesive chemotherapy application. Infected $\mathrm{T}$ cells thereafter decline to stability near zero for the rest duration of investigation.

From fig. 3(b), we illustrate the hypersurface of the effect of interplay of multiple chemotherapies $r_{1}(t)$ and $r_{2}(t)$ on both sensitive viral load and pathogen viruses. The first function $V_{s}(t)$ represents the docile behavior of the sensitive viral load with linear trajectory inclination of value $0.2 \leq V_{s}(t) \leq 1.297$ for $2 \leq t_{f} \leq 10$ months. At $19 \leq t_{f} \leq 30$ months, $V_{s}(t)$ decline to stability value $\leq 0.56$. The second function $P_{s}(t)$ exhibited similar biological progression as that of $V_{s}(t)$. This represents the sensitive pathogen with inner trajectory having value $0.1 \leq P_{s}(t) \leq 0.695$ for $2 \leq t_{f} \leq 12$ months. Reduction of $P_{s}(t)$ infection to stability is $\leq 0.28$ at the interval $19 \leq t_{f} \leq 30$ months.

Taking on fig. 3(c), we again illustrate the impact of immune effectors response under delay intracellular and boosting multiple chemotherapies. Remaining always on the positive value, the minimal decline of immune effectors response is in the range $9.489 \leq M_{E}(t) \leq 10$ for $t_{f} \leq 30$ months, which is a manifestation of the maximized $\mathrm{CD}^{+} \mathrm{T}$ cells and maximal sustainability of the CTLs with drastic suppression of $T_{s}(t), V_{s}(t)$ and $P_{s}(t)$ respectively. 
Next, we present the simulation of the costate variables as seen in fig. 4(a-c) below:

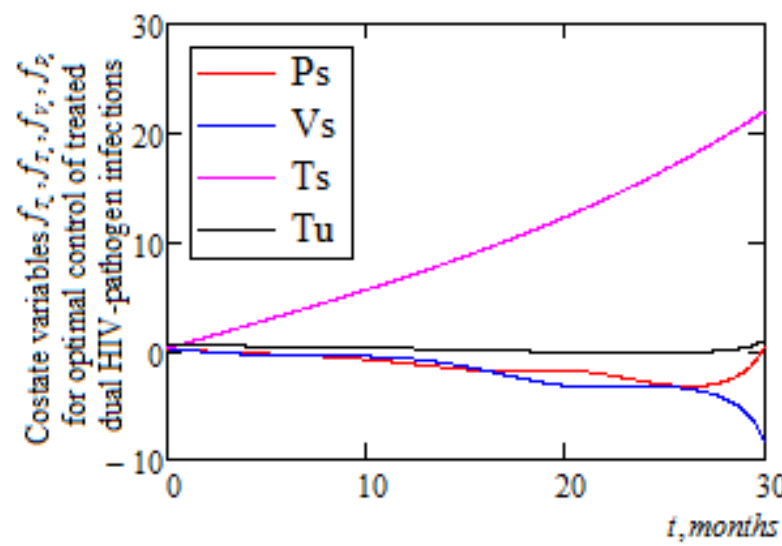

a) Simulations of costate $f_{\tau_{v}}, f_{\tau_{0}}, f_{V_{0}}, f_{v_{0}}$ with $r_{1}=0.7, r_{2}=0.3$ and $\eta_{1}=1=\eta_{2}$ for dual HIV-pathogen infections

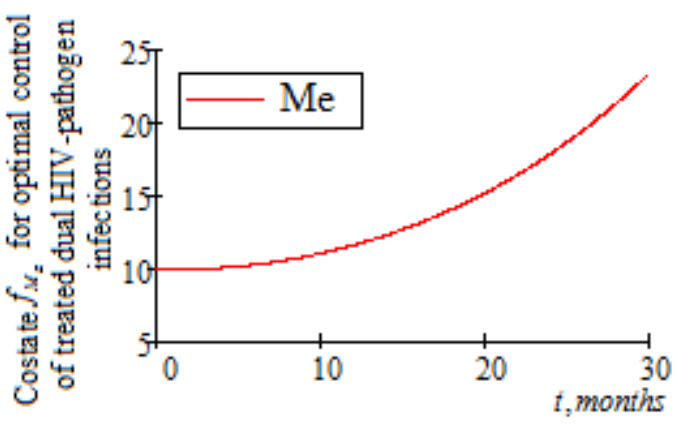

b) Simulation of costa te immune

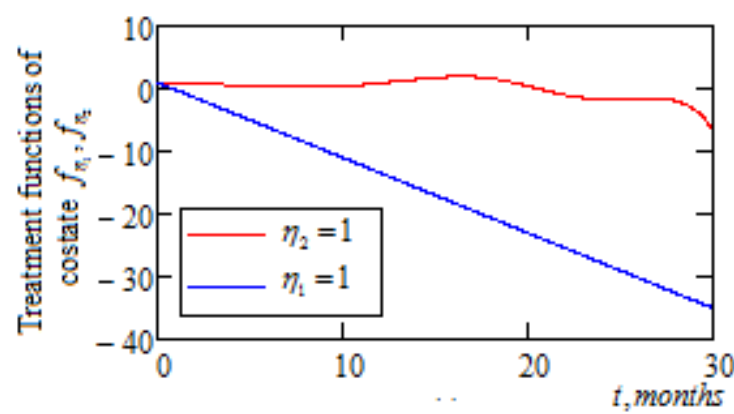

c) Simulations of costate $f_{r_{1}}, f_{r_{z}}$ for optimal control of treated dual HV-pathogen infections

Fig. 4(a-c) Simulation dynamics of costate variables for optimal treatment regiments of dual delay

$H I V$-pathogen model with $r_{1}=0.7, r_{2}=0.3$

With parameter variables as in table 2, we use fig. 4(a-c) to illustrate the concentration of uninfected cells and immune effector response in the presence of infected T-cells, sensitive virions and other costate components of the investigation. Of note, from the graphical representations of fig. 4(a-c), which shows the reverse in the increase (or decrease) as is the treated case (in fig. 3(a-c)), signifies the existence of the overall outcome of the investigation.

\section{DISCUSSION}

In the literature of this present study, we had used ordinary differential equations to seek for the determination of optimal treatment strategies for a complex nonlinear 10-Dimensional dynamic dual HIVpathogen delay-differential model. The intents of the model were to overcome the constraints of defining the critical roles of delay intracellular and immune effectors response for dual delay HIV-pathogen infections. Moreso, we formulated the model to account for the methodological application of multiple chemotherapies on incorporated dual delay HIV-pathogen infections.

Importantly, under allowable dual treatment discontinuation chambers, which suggested successive approximation processes with step-wise variations of predominant parameters till desired convergence are achieved, the model was presented as an optimal control problem with terminal time objective functional. The method of analysis explored was classical Hamilton-Pontryagin function for the evaluation of the derived system quantum locally optimal algorithm.

For simplicity and significant comparative analysis, numerical simulations for both untreated and treated cases were demonstrated along with the outcome of the co-state variables. Furthermore, the essence of enhanced treatment functions were simulated in comparison to those related prior studies of models [11, $16]$. 
Results of numerical simulations showed that treatment functions for model [11], where control variable was not introduced resulted to very large utilization in the amount of RTI and PIs required in the duration of investigation. With the introduction of control variables as was the case in [16], lesser amount of treatment functions (RTI and PIs) was required. The present model in addition to state components of model [16], further incorporated delay intracellular and immune effectors response, which completely saw to a more reduced amount of treatment functions for the entire investigation duration - see fig. 1(a-c). The consequences of uncontrolled treatment function were evaluated in fig 2(a-c). The solution of which could not guarantee the attainment of global maximum of optimized functional. The local maximum attained here, was the direct consequence of the only natural anti-HIV-pathogen, the immune effectors response.

Agreeably, the introduction of control variables as simulated by fig. 3(a-c) and affirmed by fig. 4(a-c) illustrated successive approximations of control quanta by ways of choice variations of predominant parameters. This result validated the convergence control quanta of equation (22). Of note, the inclinative parabolic trajectory of the hypersurface of $T_{u}(t)$ was a clear indication of the embedded novelty of the study when compared with those of models $[8,11,16]$.

Furthermore, the optimal drug treatment protocol led to desired maximal healthy $\mathrm{CD} 4^{+} \mathrm{T}$ cells, immune effectors and suppressed infectious viral load and pathogen. The late decline in $\mathrm{CD}^{+} \mathrm{T}$ cells suggested possible drug resistance and high presence of resistive infectious cells and virions. Finally, the never eliminated immune effectors response is arguably a function of the toxicity of the presence of dual virions infectivity. Suggestively, we note that this present quantum locally optimal algorithm for successive approximation for maximal concentration of healthy T-lymphocytes is not necessarily the only or most efficient technique but was inevitably sufficient to produce an accurate solution in an acceptable amount of drug validity period for dual delay HIV-pathogen infections.

\section{CONCLuSion}

We present a necessary and sufficient quantum locally optimal algorithm for successive approximation technique, which produced an accurate solution in an acceptable amount of drug validity period for the treatment of dual delay HIV-pathogen infections. The model was formulated as an optimal control problem structured on a nonlinear complex 10-Dimensonal mathematical differential model with terminal time objective functional. The result of numerical simulations not only affirmed the maximization of healthy T-lymphocytes and maximal sustained cytotoxic T-lymphocytes as a function of high intensity of successive iterations of multiple chemotherapy, which were more rewarding at onset of infection but is sufficiently attributed to the immense critical role of incorporated delay intracellular and boosted natural adaptive immune effectors response. Furthermore, the enhanced significant reduction in systemic cost was a function of delay intracellular, which accounted for intermittent chemotherapy discontinuation. Decline in healthy $\mathrm{CD}^{+} \mathrm{T}$ cells suggests systemic discontinuation or change in chemotherapy administration. Therefore, the study advocates for a more articulated mathematical model and medical innovations that could address the persistent resistive infectious components, which were a thwarting factors.

\section{ACKNOWLEDGMENTS}

My utmost appreciation to the anonymous referee(s) for his/her valuable comments on the first version of the manuscript, which have led to an improvement in this revised version.

\section{REFERENCES}

[1] Hattaf, K. and Yousfi, N., Optimal Control of a Delayed HIV Infection Model with Immune Response Using an Efficient Numerical Method, Biomathematics, 1-7, (2012).

[2] Fister, K. R., Lenhart, S. and McNally, J. S., Optimizing chemotherapy in an HIV Model,Electr. J. Diff. Eq., 32, 1-12, (1998).

[3] Bassey E., Discretization method for optimization control model for treatment of pathogenic induced HIV infection. Curr. Trends Clin. Med. Imaging, 1(5): 555571. 1-6, (2017).

[4] Joshi, H. R., Optimal Control of an HIV Immunology Model. Optimal Control Applications and Methods, 23: 199-213, (2002). 
[5] Adams, B. M., Banks, H.T., Hee-Dae K. and Tran, H.T., Dynamic Multidrug Therapies for HIV: Optimal and STI Control Approaches,(2004). http://citeseerx.ist.psu.edu/viewdoc/summary?doi=10.1.1.400.9056 . Accessed May 28, 2017.

[6] Bassey, E.B., Lebedev, K. A., On Analysis of Parameter Estimation Model for the Treatment of PathogenInduced HIV Infectivity, Open Access Library Journal, 3(4): 1-13, (2016).

[7] Bassey, B. E. and Andreyevich, L. K., On Quantitative Approach to Parametric Identifiability of Dual HIV Parasitoid Infectivity Model,Open Access Library Journal, 3: e2931, 1-14, (2016).

[8] Bassey, E. B., Kimbir, R. A. and Lebedev, K. A., On Optimal Control Model for the Treatment of Dual HIVParasitoid Pathogen Infection, J Bioengineer \& Biomedical Sci., 7: 212, 1-7, (2016)

[9] Bassey E. B., Optimal Control Model for Pair Chemotherapy Treatment with Time-delay Immunity in Dual HIV-Infectivity,Applied Mathematics and Physics, 5, 2, 61-76, (2017).

[10] Marcluck, G. I., Methods of numerical mathematics, $2^{\text {nd }}$ ed. (Nauka, Moscow, 1980) Springer, New York, (1975).

[11] Kirschner, D. E. and Webb, G. F., Resistance, Remission, and Qualitative Differences in HIV Chemotherapy, Emerging Infectious Diseases, 3(3), 273-283, (1997). https://dx.doi.org/10.3201/eid0303.970303

[12] Butler S., Kirschner, D. and Lenhart S., Optimal control of the chemotherapy affecting the infectivity of HIV, Editors: O. Arino, D. Axelrod and M. Kimmel,Advances in Mathematical Population Dynamics- Molecules, Cells and Man, 557-569, (1997).

[13] Kirschner, D., Lenhart, S., Serbin, S., Optimal control of the chemotherapy of HIV, Journal of Mathematical Biology, 35, 775-792, (1997).

[14] Stengel, R.F., Ghigliazza, R., Kulkarni, N. and Laplace, O., Optimal control of innate immune response, Optimal Contr. Appl. Methods, 23, 91-104, (2002).

[15] Shudo, E. and Iwasa, Y., Dynamic Optimization of Host Defense, Immune Memory, and Post-Infection Pathogen Levels in Mammals, J. Theor. Biol., 228, 17-29, (2004).

[16] Velichenko, V.V. and Pritykin, D. A., Numerical Methods of Optimal Control of the HIV-Infection Dynamics,Journal of Computer and Systems Sciences International, 45, No. 6, 894-905, (2006).

[17] Adams, B. M., Banks, H. T., Davidian, M., Kwon, Hee-Dae, Tran, H. T., Wynne, S. N. and Rosenberg, E.S., HIV Dynamics: Modeling, data analysis, and optimal treatment protocols,J. Comp. Appl. Math., 184, 10-49, (2005). http://citeseer.ist.psu.edu/viewdoc/summary?doi=10.1.1.115.8107 . Accessed May 28, 2017.

[18] Buldaev, A. S. and Pogozhev, I. B.,Optimization of Immune Process According to the Criterion of Minimum of the Index of Illness Severity,VTs SO AN SSSR, Novosibirsk, (1986). [in Russian].

[19] Velichenko, V. V., A numerical method for solving optimal control problems. Zh. Vychisl. Mat. Mat. Fiz., 6, 4, 635-647, (1966).

[20] Pontryagin, L. S., Boltyanskii, V. G., Gamkrelidze, R.V. and Mishchenko, E. F., The Mathematical Theory of Optimal Processes, vol. 4, Gordon \& Breach Science Publishers, New York, NY, USA, (1986).

\section{AUTHOR'S BIOGRAPHY}

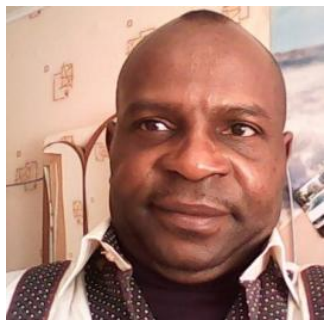

Bassey Echeng Bassey, a $\mathrm{PhD}$ holder in Computational Mathematics and Informatics from Kuban State University, Krasnodar Russia and M.Sc. in Industrial Mathematics, Federal University of Agriculture, Makurdi, Nigeria. He is a lecturer and presently the Co-coordinator - Head of Pre-degree program, Cross River University of Technology, Calabar, Nigeria. Published more than 21 papers in reputed journals, monographs' and author of textbooks. Research interests include but not limited to: Mathematical modeling, numerical methods; simulation analysis, mathematical methods, computer technology and programming.

Citation: B. E. Bassey, "Quantum Optimal Control Dynamics for Delay Intracellular and Multiple Chemotherapy Treatment (MCT) of Dual Delayed HIV - Pathogen Infections ", International Journal of Scientific and Innovative Mathematical Research, vol. 5, no. 6, p. 1-19, 2017., http://dx.doi.org/10.20431/2347-3142.0506001

Copyright: () 2017 Authors. This is an open-access article distributed under the terms of the Creative Commons Attribution License, which permits unrestricted use, distribution, and reproduction in any medium, provided the original author and source are credited. 